\title{
PDS5B inhibits cell proliferation, migration, and invasion via upregulation of LATS1 in lung cancer cells
}

\author{
Hui Xu ${ }^{1,7}$, Wenjing Zhou ${ }^{2,7}$, Fan Zhang ${ }^{3}$, Linhui $\mathrm{Wu}^{2}$, Juan $\mathrm{Li}^{2}$, Tongtong Ma ${ }^{4}$, Tong Cao ${ }^{5}$, Chaoqun Lian ${ }^{6}$, Jun Xia ${ }^{6}$, Peter Wang $\mathbb{D}^{6 凶}$, \\ Jia Ma ${ }^{6{ }^{\prime}}$ and Yuyun $\mathrm{Li}^{1{ }^{\boxplus}}$
}

(c) The Author(s) 2021

PDS5B (precocious dissociation of sisters 5B) plays a pivotal role in carcinogenesis and progression. However, the biological functions of PDS5B in lung cancer and its underlying mechanisms are not fully elucidated. In the present study, we used MTT assays, wound-healing assays, and transwell migration and invasion approach to examine the cell viability, migration, and invasion of non-small cell lung cancer (NSCLC) cells after PDS5B modulation. Moreover, we investigated the function of PDS5B overexpression in vivo. Furthermore, we detected the expression of PDS5B in tissue samples of lung cancer patients by immunohistochemical study. We found that upregulation of PDS5B repressed cell viability, migration, and invasion in NSCLC cells, whereas downregulation of PDS5B had the opposite effects. We also observed that PDS5B overexpression retarded tumor growth in nude mice. Notably, PDS5B positively regulated LATS1 expression in NSCLC cells. Strikingly, low expression of PDS5B was associated with lymph node metastasis in lung cancer patients. Our findings suggest that PDS5B might be a therapeutic target for lung cancer.

Cell Death Discovery (2021)7:168; https://doi.org/10.1038/s41420-021-00537-6

\section{INTRODUCTION}

Lung cancer is the most common tumor and is the first leading cause of mortality in the United States [1]. It will be expected that 228,820 new patients with lung cancer and 135,720 deaths for this disease will be happened in the United States in 2020 [1]. Approximately $25 \%$ of all cancer deaths are because of lung cancer, and the 5-year relative survival rate of lung cancer is $19 \%$ [1]. Fifty-seven percent of patients with lung cancer have metastasis when they were diagnosed, leading to a $5 \%$ survival rate for these metastatic lung cancer patients [2]. The treatment strategies include surgery, chemotherapy, immunotherapy, and combination therapies [3-5]. Although the therapies have been improved, the outcomes of lung cancer patients are still not satisfactory. Therefore, it is necessary to discover new biomarkers for the prediction of early-stage lung cancer and to find novel targets for the treatment of lung cancer.

Evidence has demonstrated that several genes play an important role in lung cancer development and malignant progression. For example, the epidermal growth factor receptor often has mutations in lung adenocarcinoma [6]. Moreover, microRNAs, long non-coding RNA (IncRNAs), and circular RNAs (circRNAs) are critically involved in lung tumorigenesis [7-11]. Hippo signaling pathway has been identified to participate in lung tumor initiation and progression [12-14]. It has been known that Hippo pathway negatively controls the expression of YAP and TAZ, two transcriptional co-activators. This pathway consists of several kinases such as mammalian STE20-like protein kinase 1 (MST1), MST2, large tumor suppressor 1 (LATS1), and LATS2. MST1/ 2 activates LATS1/2 and MOB1A/1B via phosphorylation, and LATS1/2 activates YAP and TAZ phosphorylation, leading to inhibition of YAP and TAZ activation [15, 16].

Precocious dissociation of sisters 5B (PDS5B), also known as APRIN, was reported to participate in carcinogenesis. Knockdown of PDS5B disrupts stem cell programs by regulating Oct4, Nanog, and SOX2 patterns in embryonal carcinoma [17]. One study revealed that PDS5B expression is correlated with histological grade in breast cancer and the treatment efficacy of chemotherapy in breast cancer patients [18]. PDS5B, as a BRCA2-interacting protein, is required for DNA repair and genome integrity in breast cancer cells [18]. Another study demonstrated that miR-27a represses the expression of PDS5B in prostate cancer cells, leading to the promotion of cell viability [19]. PDS5B exhibits frameshift mutations in gastric cancer and colorectal cancer patients, which is associated with weak or negative PDS5B immunostaining [20]. In addition, a frameshift mutation of PDS5B is observed in breast cancer tissues and lymph nodes [21]. PDS5B is highly expressed in oral squamous cell carcinomas, but PDS5B is not correlated with the expression of proliferation cell markers such as p53 and ki-67 [22]. The functions and role of PDS5B in lung cancer cells have not been fully elucidated. Therefore, in this study, we explored the biological functions of PDS5B in lung cancer cells and determined its underlying molecular mechanism.

\footnotetext{
${ }^{1}$ Department of Laboratory Medicine, School of Laboratory Medicine, Bengbu Medical College, Bengbu, Anhui 233030, China. ${ }^{2}$ Bengbu Medical College Key Laboratory of Cancer Research and Clinical Laboratory Diagnosis, Bengbu Medical College, Bengbu, Anhui 233030, China. ${ }^{3}$ Department of Pharmacology, Adagene Limited Company, Suzhou, Jiangsu 215000, China. ${ }^{4}$ School of Laboratory Medicine, Bengbu Medical College, Bengbu, Anhui 233030, China. ${ }^{5}$ Department of Clinical Laboratory, The First Affiliated Hospital of Bengbu Medical College, Bengbu, Anhui 233004, China. ${ }^{6}$ Department of Biochemistry and Molecular Biology, School of Laboratory Medicine, Bengbu Medical College, Bengbu, Anhui

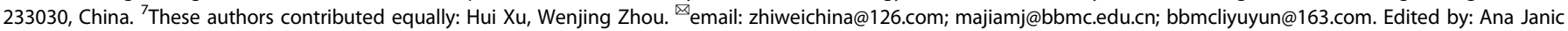




\section{RESULTS}

\section{PDS5B overexpression inhibits cell viability}

The expression levels of PDS5B in the panel of lung cancer cells and the normal lung epithelial BEAS-2B cells were measured by western blotting. We found that PDS5B is highly expressed in BEAS-2B cells (Fig. 1A). To determine the role of PDS5B in lung cancer cells, PDS5B expression was downregulated by siRNA transfection in non-small cell lung cancer (NSCLC) cells. Our Western blotting data demonstrated that PDS5B expression was downregulated in both $\mathrm{H} 1975$ and $\mathrm{H} 460$ cell lines (Fig. 1B). Moreover, we also transfected PDS5B cDNA into NSCLC cells via Lipofectamine 3000. We observed that PDS5B expression was elevated in $\mathrm{H} 1975$ and $\mathrm{H} 460$ cells after CDNA transfection (Fig. 1C). Next, we explored whether PDS5B modulation affected the viability of NSCLC cells by MTT assays. The results from our MTT assays revealed that downregulation of PDS5B promoted cell viability, whereas overexpression of PDS5B repressed the viability of NSCLC cells (Fig. 1D).
A

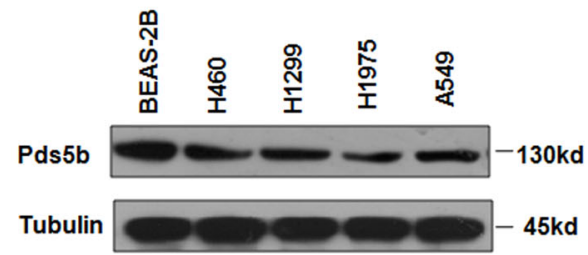

B

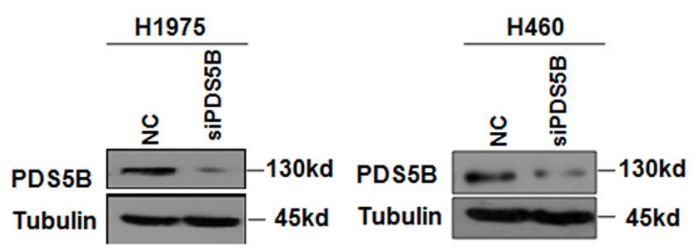

C
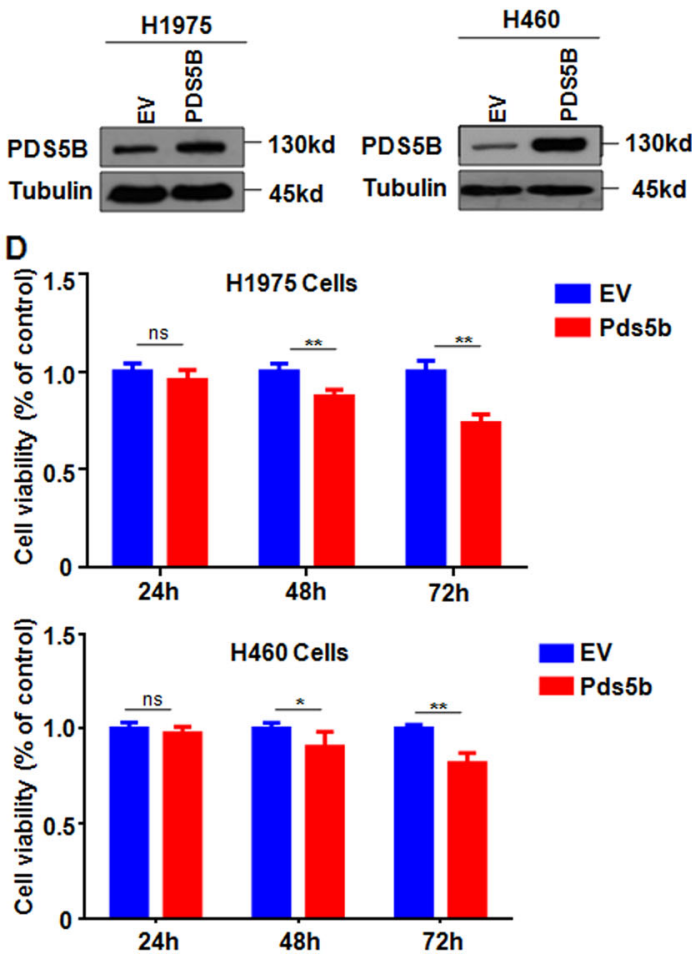
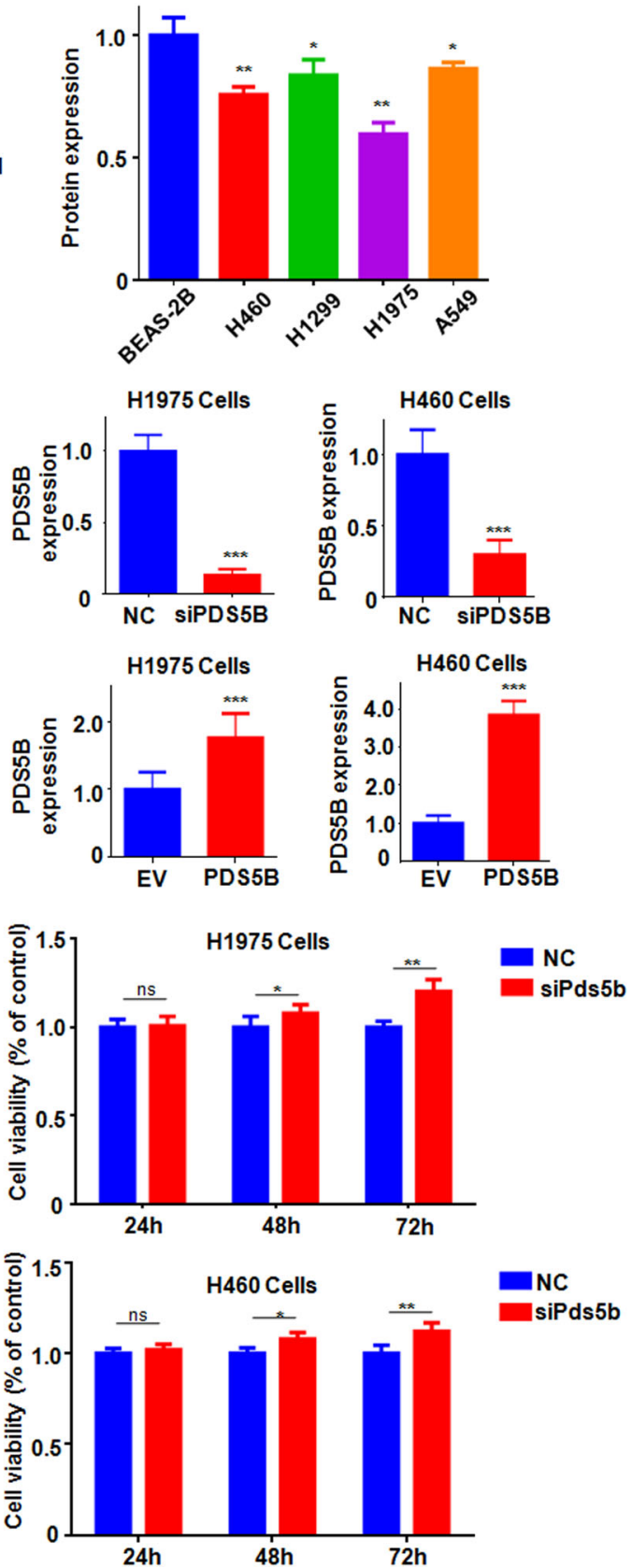

Fig. 1 PDS5B overexpression represses cell viability. A Left panel: the expression of PDS5B was measured in the panel of lung cancer cells and the normal lung epithelial BEAS-2B cells. Right panel: Quantitative results of PDS5B expression. ${ }^{* *} P<0.01$ vs BEAS-2B cells. B Left panel: The expression of PDS5B was decreased in NSCLC cells after PDS5B siRNA transfection. Right panel: quantitative results of PDS5B expression. ${ }^{* * *} P<0.001$ vs NC group. NC nonspecific control siRNA, siPDS5B PDS5B siRNA. NC group is the control group. C Left panel: the expression of PDS5B was increased in NSCLC cells after PDS5B CDNA transfection. Right panel: quantitative results of PDS5B expression. ${ }^{* * * P}<0.001$ vs EV group. EV empty vector, PDS5B PDS5B cDNA. EV group is the control group. D Effects of PDS5B modulation on cell viability at different times in NSCLC cells were examined by MTT assays. ${ }^{* *} P<0.01$ vs control group. NC nonspecific control siRNA, siPDS5B PDS5B siRNA. EV empty vector, PDS5B PDS5B CDNA. All data are representative of three independent experiments. Data are shown as mean \pm SD from experiments in triplicate. 
PDS5B overexpression suppresses cell migration and invasion To elucidate whether PDS5B could affect migration and invasion of lung cancer cells, wound-healing assays were performed in $\mathrm{H} 1975$ and $\mathrm{H} 460$ cells after PDS5B modulation. We found that overexpression of PDS5B delayed the wound closure in $\mathrm{H} 1975$ and H460 cells, indicating that PDS5B upregulation repressed cell migration (Fig. 2A). In line with this finding, downregulation of PDS5B promoted wound closure in NSCLC cells (Fig. 2B). To further validate the role of PDS5B in controlling migratory capacity, transwell chamber migration assays were conducted in NSCLC cells after PDS5B changes. Overexpression of PDS5B reduced the migrated cell numbers in $\mathrm{H} 1975$ and $\mathrm{H} 460$ cells (Fig. 2C). Moreover, transwell invasion assay data demonstrated that PDS5B overexpression decreased the invaded cell numbers through the membrane with coating Matrigel (Fig. 2C). Consistently, PDS5B knockdown promoted cell migratory and invasive capacities in NSCLC cells (Fig. 2C). Taken together, PDS5B governs the migration and invasion of NSCLC cells.

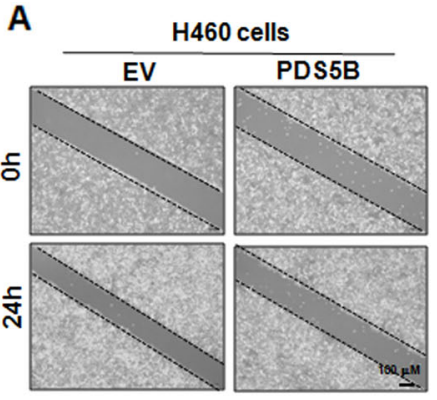

B

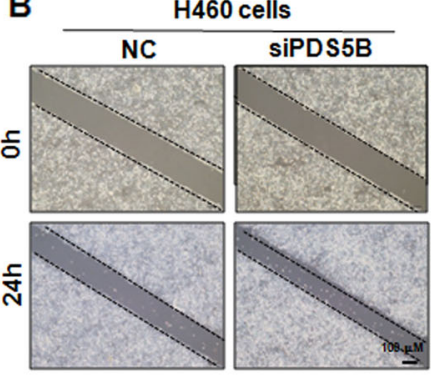

C

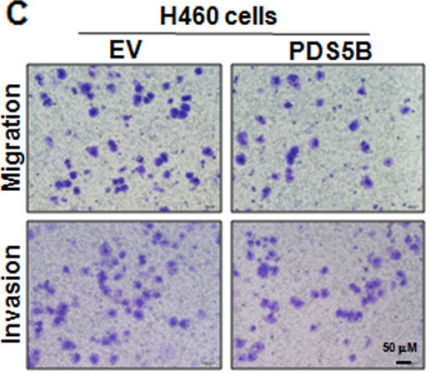

D

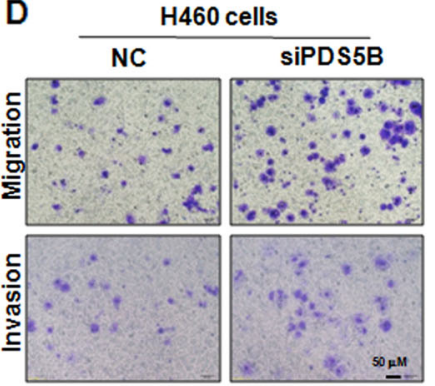

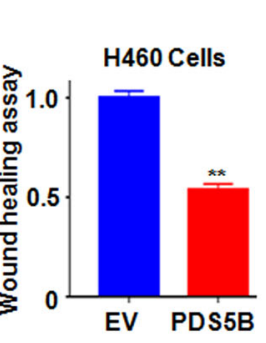
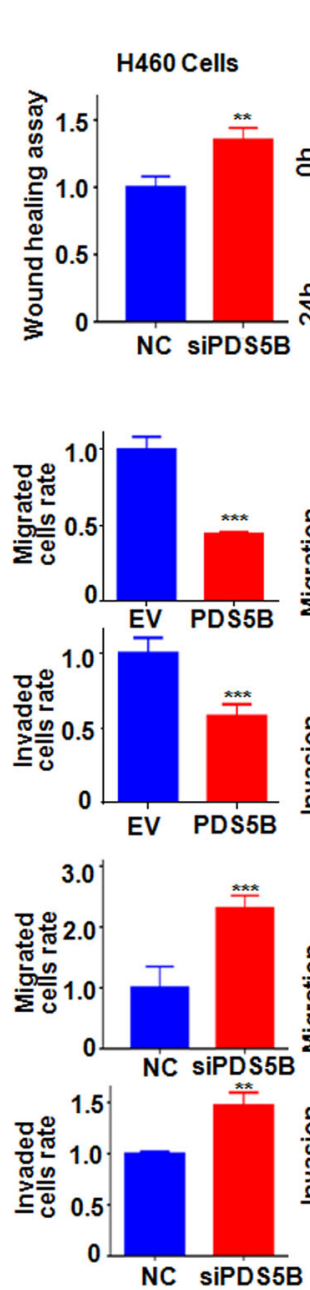
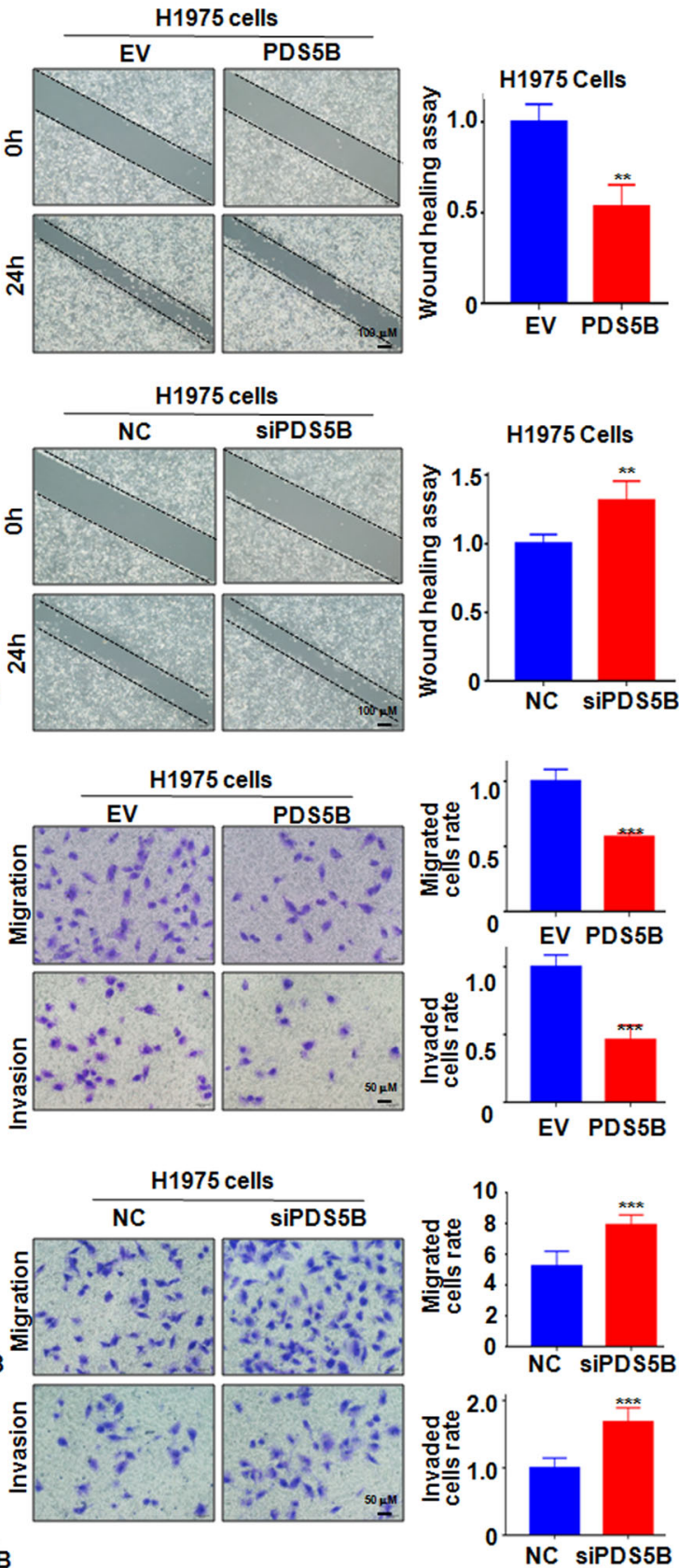

Fig. 2 PDS5B overexpression retards cell motility. A Left panel: wound closure was examined by wound-healing assays in NSCLC cells after PDS5B cDNA transfection. Right panel: quantitative data of wound closure. ${ }^{*} P<0.01$ vs EV group. EV empty vector, $P D S 5 B$ PDS5B cDNA. EV group is the control group. B Left panel Wound closure was detected by wound-healing assays in NSCLC cells after PDS5B siRNA transfection. Right panel: quantitative data of wound closure. ${ }^{* *} P<0.01$ vs NC group. NC nonspecific control siRNA, siPDS5B PDS5B siRNA. NC group is the control group. C Left panel cell motility was examined in NSCLC cells after PDS5B cDNA transfection. Right panel: quantitative data of cell motility. ${ }^{* * *} P<0.001$ vs EV group. EV group is the control group. D Left panel: cell motility was measured in NSCLC cells after PDS5B siRNA transfection. Right panel: quantitative data of cell motility. ${ }^{* *} P<0.01,{ }^{* * *} P<0.001$ vs NC group. NC group is the control group. Data are shown as mean \pm SD from experiments in triplicate. 
A
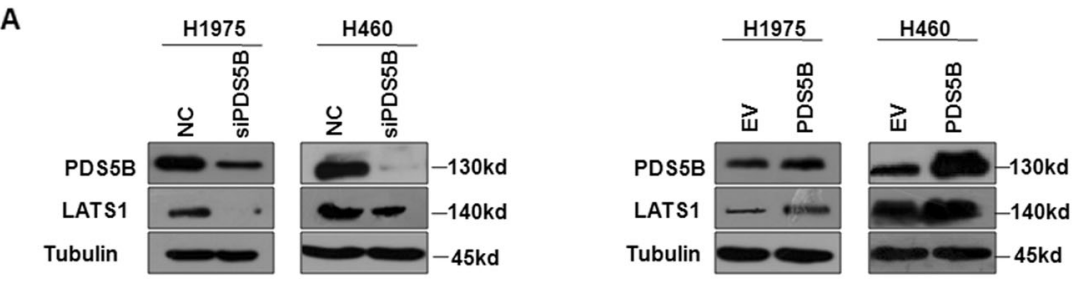

B
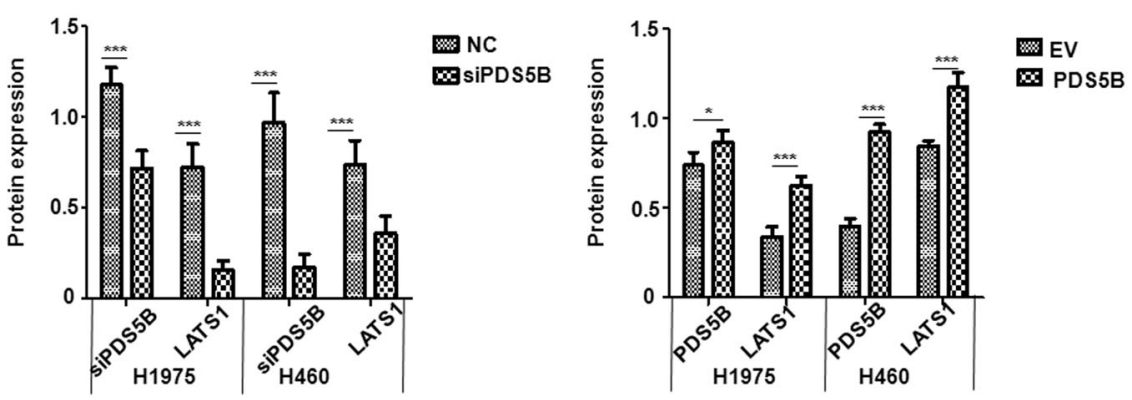

Fig. 3 PDS5B regulates LATS1 expression. A The expression of PDS5B and LATS1 was examined by western blotting in $\mathrm{H} 1975$ and $\mathrm{H} 460$ cells after PDS5B modulation. EV empty vector, PDS5B PDS5B cDNA. NC nonspecific control siRNA, siPDS5B PDS5B siRNA. NC and EV groups are the control groups. B Quantitative data of western blotting results. ${ }^{*} P<0.05,{ }^{* *} P<0.001$ vs control group. Data are shown as mean \pm SD from experiments in triplicate.

A
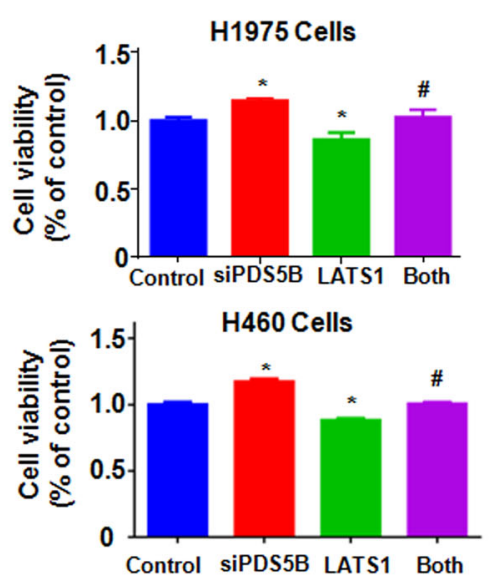

B
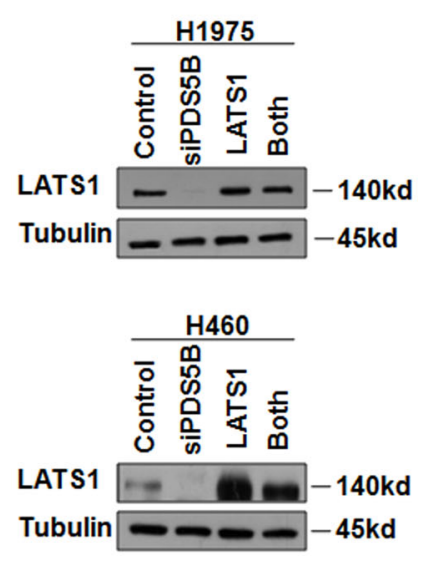

C
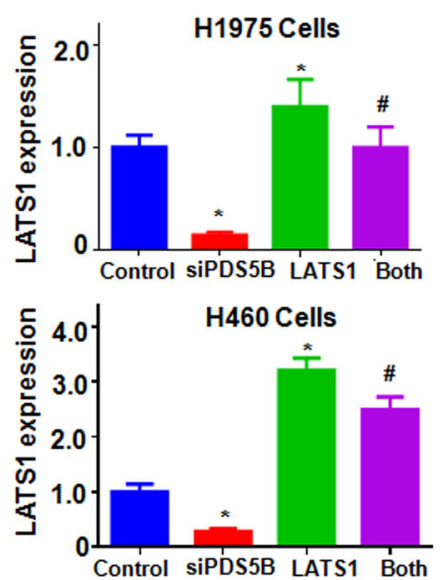

Fig. 4 Overexpression of LATS1 rescues PDS5B knockdown-induced cell viability. A Cell viability was determined by MTT assays in NSCLC cells after co-transfection of LATS1 cDNA and PDS5B siRNA. ${ }^{* *} P<0.05$ vs control group; ${ }^{\#} P<0.05$ vs PDS5B siRNA alone or LATS1 CDNA alone. Control nonspecific control siRNA and empty vector, siPDS5B PDS5B siRNA, LATS1 LATS1 cDNA, Both: siPDS5B + LATS1. Data are shown as mean \pm SD from experiments in triplicate. B The expression of LATS1 was examined in NSCLC cells after co-transfection of LATS1 cDNA and PDS5B siRNA. C Quantitative data of LATS1 expression. Data are shown as mean \pm SD from experiments in triplicate.

\section{PDS5B overexpression increases LATS1 expression}

To determine the underlying mechanism of PDS5B-mediated antitumor activity in NSCLC cells, we measured the expression of LATS1 in H1975 and H460 cells after PDS5B modulation by western blotting analysis. Our data uncovered that PDS5B upregulation elevated the expression of LATS1 in H1975 and H460 cells, whereas PDS5B silencing alleviated the LATS1 expression levels in NSCLC cells (Figs. 3A, B). These results suggest that PDS5B positively regulates the LATS1 expression.

\section{Overexpression of LATS1 rescues PDS5B knockdown-induced cell viability}

To dissect whether PDS5B could govern cell viability via regulating LATS1 pathway, we overexpressed LATS1 in NSCLC cells after PDS5B downregulation. Overexpression of LATS1 reduced the viability of $\mathrm{H} 1975$ and $\mathrm{H} 460$ cells (Fig. 4A). Importantly, LATS1 overexpression abolished the promotion of cell viability that was induced by the knockdown of PDS5B in both NSCLC cell lines (Fig. $4 A$ ). Western blotting analysis data showed that upregulation of LATS1 abrogated the PDS5B knockdown-mediated inhibition of LATS1 in NSCLC cells (Figs. 4B, C).

\section{LATS1 overexpression abrogates PDS5B knockdown-mediated motility}

Next, we performed wound-healing assays in NSCLC cells after PDS5B knockdown in combination with LATS1 overexpression. Upregulation of LATS1 delayed wound closure in H1975 and H460 cells (Fig. 5A). Moreover, overexpression of LATS1 rescued PDS5B siRNA-mediated enhancement of wound closures in H1975 and H460 cells (Fig. 5A). Furthermore, LATS1 upregulation suppressed migration and invasion of H1975 and H460 cells by transwell chamber assays (Fig. 5B). Strikingly, 
A

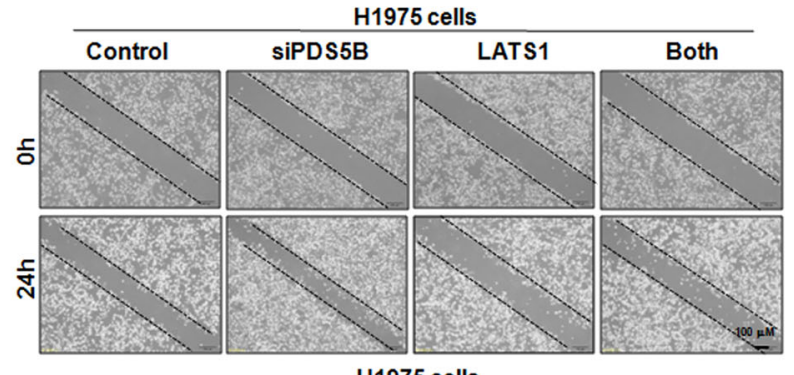

B
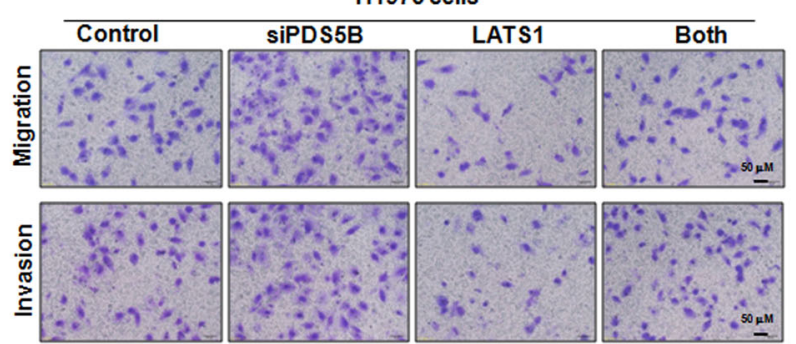

C
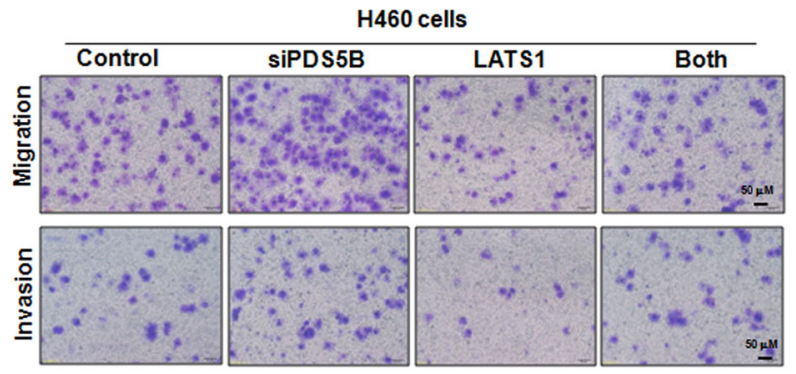
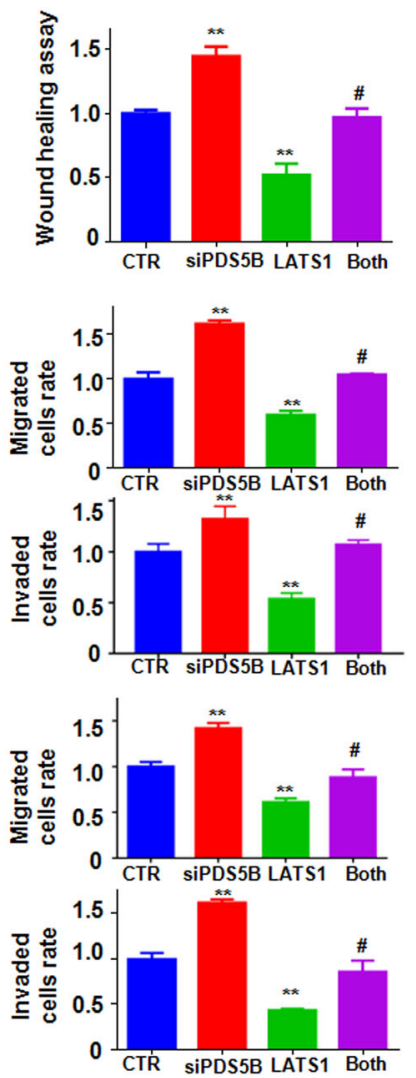

Fig. 5 LATS1 overexpression abrogates PDS5B knockdown-mediated motility. A Left panel: wound closure was examined by woundhealing assays in H1975 cells after co-transfection of LATS1 cDNA and PDS5B siRNA. Right panel: quantitative data of wound closure. ${ }^{* *} P<0.05$ vs control group; ${ }^{\#} P<0.05$ vs PDS5B siRNA alone or LATS1 CDNA alone. Control nonspecific control siRNA and empty vector, siPDS5B PDS5B siRNA, LATS1 LATS1 cDNA, Both siPDS5B + LATS1. B Left panel: cell motility was examined in H1975 cells after co-transfection of LATS1 cDNA and PDS5B siRNA. Right panel: quantitative data of cell motility. ${ }^{* *} P<0.05$ vs control group; ${ }^{*} P<0.05$ vs PDS5B siRNA alone or LATS1 cDNA alone. C Left panel: cell motility was measured in $\mathrm{H} 460$ cells after co-transfection of LATS1 CDNA and PDS5B siRNA. Right panel: Quantitative data of cell motility. ${ }^{* *} P<0.05$ vs control group; ${ }^{\#} P<0.05$ vs PDS5B siRNA alone or LATS1 cDNA alone. Data are shown as mean \pm SD from experiments in triplicate.

overexpression of LATS1 abolished PDS5B silencing-induced migration and invasion in two NSCLC cell lines (Fig. 5B). Thus, PDS5B inhibited cell motility partly via upregulation of LATS1 in NSCLC cells.

\section{Overexpression of PDS5B suppresses tumor growth in vivo}

To determine whether PDS5B could retard tumor growth in vivo, we performed a mouse study using lung cancer xenografts in nude mice. We found that PDS5B overexpression reduced tumor growth compared with the control group (Fig. 6A, B). The tumor weights and volumes were less in the PDS5B-overexpressing group (Fig. 6A, B). Furthermore, the expression of PDS5B and LATS1 was determined in mouse tumor tissues by western blotting. We observed that LATS1 expression was increased in the PDS5B-overexpressing group (Fig. 6C).

PDS5B expression is associated with lymph node metastasis To ascertain the expression of PDS5B in tumor specimens of lung cancer patients, we used immunohistochemical staining to detect PDS5B expression levels in tumor tissues of 232 patients with lung cancer. PDS5B was detected to be expressed in 39 tumor tissues, and 193 patients displayed no detectable expression of PDS5B. Moreover, low expression of PDS5B was associated with lymph node metastasis in lung cancer patients. In addition, $70 \%$ of lung cancer patients exhibited undetectable or lower expression of LATS1. Notably, the expression of PDS5B was correlated with LATS1 expression levels in tumor specimens in lung cancer patients (Fig. 6D).

\section{DISCUSSION}

PDS5B is an important factor for proper cohesion dynamics and protection of replication fork [23]. The Shu complex, which consists of SWS1 and SWSAP1, interacts with PDS5B and SPIDR and governs DNA repair [24]. PDS5B has been reported to involve in tumorigenesis and tumor progression. For instance, low expression of PDS5B is associated with better survival in patients with ovarian cancer [25]. In the current study, low expression of PDS5B was observed and correlated with lymph node metastasis in lung cancer patients, indicating that PDS5B might be associated with the prognosis of lung cancer patients. One study revealed that PDS5B exerts antitumor activity in pancreatic cancer cells [26]. Overexpression of PDS5B represses cell viability and stimulates apoptosis in pancreatic cancer cells, while depletion of PDS5B increases migratory and invasive ability [26]. In support of the tumor-suppressive role of PDS5B, our present study demonstrated that PDS5B upregulation repressed viability, migration, and invasion of lung cancer cells, while PDS5B knockdown promoted viability and motility of NSCLC cells. Strikingly, PDS5B upregulation 
A

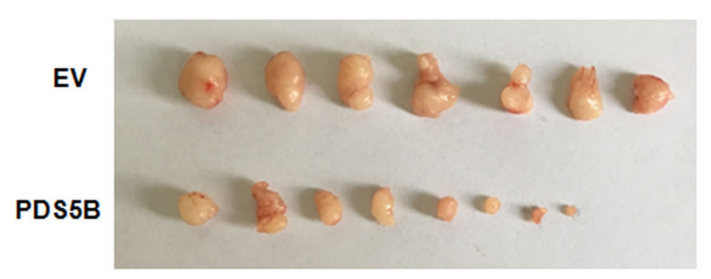

B
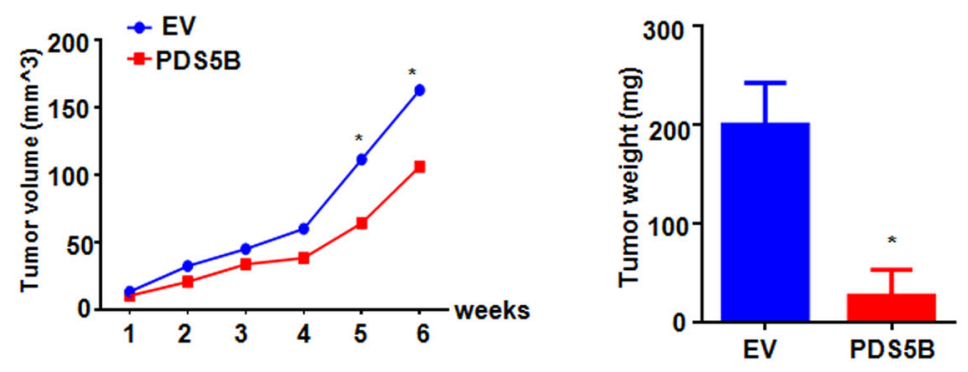

C
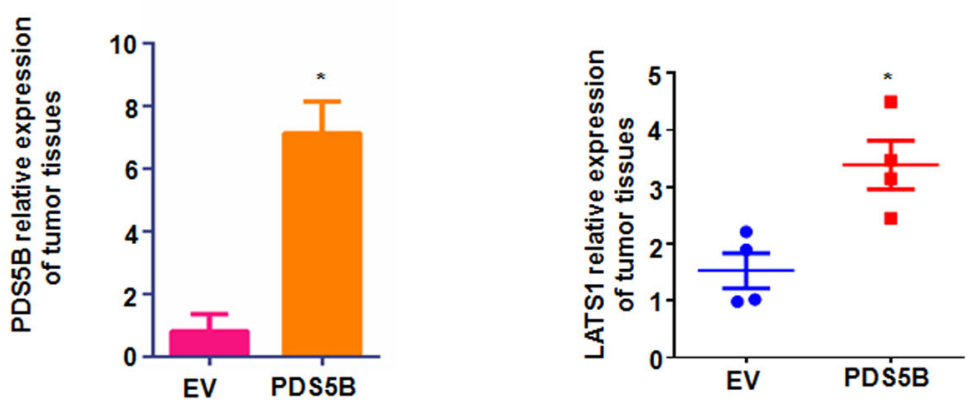

D

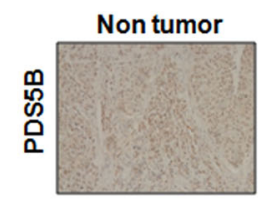

Non tumor

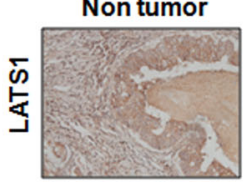

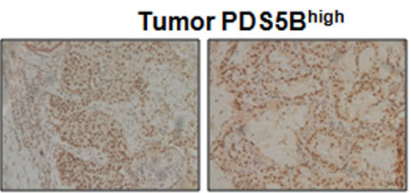

Tumor LATS 1 high
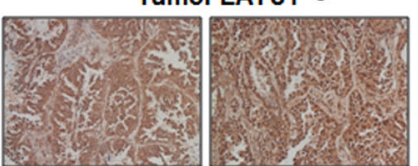

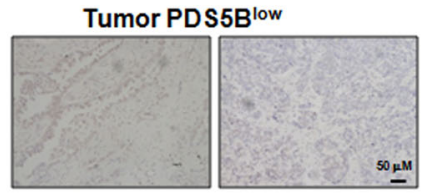

Tumor LATS1low

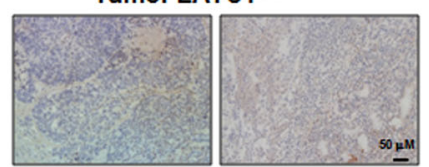

Fig. 6 Overexpression of PDS5B suppresses tumor growth in vivo. A. The tumors in nude mice were dissected and taken a picture. EV empty vector, PDS5B PDS5B CDNA. EV group is the control group. B In vivo tumor growth was measured per week and the weights of the dissected tumors. Data are shown as mean \pm SD. ${ }^{*} P<0.05$ vs EV group. $C$ The expression of PDS5B and LATS1 in tissues from xenografted tumors. Data are shown as mean \pm SD. ${ }^{*} P<0.05$ vs EV group. D Immunohistochemical staining images of PDS5B and LATS1 in lung cancer tissues.

retarded tumor growth in vivo, further supporting the anticancer role of PDS5B in lung cancer.

PDS5B alleviates cell viability, migration, and invasion via promoting Ptch2 expression in pancreatic cancer cells [27]. Interestingly, one group reported that PD-L1 competes with WAPL to bind to PDS5B and performs tumor progression independent of its role in the immune checkpoint [28]. Moreover, silencing of PDS5B expression promotes cell proliferation via induction of IL-6 secretion and activation of STAT3, and promotion of cyclin D1 expression in human cancer [29]. In the present study, we reported that PDS5B positively regulated the expression of LATS1 in lung cancer cells. Notably, overexpression of LATS1 abrogated PDS5B knockdown-induced tumor promotion in NSCLC cells, suggesting that LATS1 is involved in PDS5B-mediated anticancer activity in lung cancer. In line with this concept, PDS5B was associated with the expression of LATS1 in tumor tissues of lung cancer patients.

It has been reported that miR-223 promotes the viability and motility of pancreatic cancer cells via inhibition of PDS5B in pancreatic cancer cells [26]. It is required to investigate whether miR-223 or other miRNAs target PDS5B expression in lung cancer cells, which will help us understand the regulatory mechanism of PDS5B. Downregulation of PDS5B increased the sensitivity of the PARP inhibitor Olaparib, indicating that PDS5B is a potential target for treating ovarian cancer [25]. It is necessary to determine whether upregulation of PDS5B could enhance the sensitivity of chemotherapeutic drugs in lung cancer. As PDS5B might be a useful target for fighting cancer, it is pivotal to discover the compounds to modulate the expression of PDS5B and to obtain a better treatment outcome for cancer patients. 


\section{MATERIALS AND METHODS}

\section{Cell culture}

Human lung epithelial BEAS-2B cells were kindly provided by EK-Bioscience Company (Shanghai, China). Human non-small-cell lung cancer cell lines (H1975 and H460 cells) were cultured in Roswell Park Memorial Institute Medium (RPMI)-1640 medium, supplemented with 10\% fetal bovine serum (FBS, Gibco, USA) and $1 \%$ streptomycin and penicillin in a humidified atmosphere containing $5 \% \mathrm{CO}_{2}$ at $37^{\circ} \mathrm{C}$.

\section{Transfection}

The H1975 and H460 cells were grown in six-well plates and transfected with PDS5B CDNA or PDS5B siRNA or nonspecific control siRNA or empty vector using Lipofectamine 3000 according to manufacturer's protocol [30]. The PDS5B siRNAs were purchased from GenePharma Company (Shanghai, China).

\section{MTT assays}

The H1975 and H460 cells were grown in 96-well plates. After overnight incubation, NSLC cells were transfected with PDS5B CDNA or PDS5B siRNA for 24,48 , and $72 \mathrm{~h}$. Then, MTT assay was performed as described before [31]. In brief, the transfected cells were harvested and MTT agent $(50 \mu \mathrm{L})$ was incubated to each well for $2 \mathrm{~h}$. Dimethyl sulfoxide was used to dissolve formazan cells after the supernatant was removed. The OD value at $590 \mathrm{~nm}$ was measured by a microplate reader for detecting cell viability.

\section{Wound-healing assays}

The transfected lung cancer cells were grown in six-well plates and incubated at $37{ }^{\circ} \mathrm{C}$. When the cells obtained $90 \%$ of confluence, the wound scrape was created by a $100 \mu \mathrm{l}$ sterile pipette tip. The supernatant cells were washed away with PBS. The scratched areas were imaged at $0 \mathrm{~h}$ and $24 \mathrm{~h}$, respectively [31]. The distances between gaps were calculated.

\section{Transwell migration and invasion assays}

The 24-well plate with Transwell chambers was utilized for detecting migration and invasion. The transfected cells with RPMI-1640 medium without FBS were seeded into the upper inserts. RPMI-1640 medium with FBS was added into the lower chamber. Non-migrated cells or non-invaded cells were removed by cotton swabs after $24 \mathrm{~h}$. The migrated and invaded cells were stained with Crystal Violet. The images were captured by a microscope as described before [32].

\section{Western blotting analysis}

H1975 and H460 cells were harvested after PDS5B modulation and total proteins from cells were extracted. The expression of PDS5B and LATS1 was measured by Western blotting analysis as described previously [31]. The protein from cell lysate was separated by sodium dodecyl-sulfate polyacrylamide gel electrophoresis and transferred to the polyvinylidene difluoride membrane. Then, the membranes were incubated with antiPDS5B, anti-LATS1, or anti-tubulin antibodies. After washed by TBST three times, the membranes were incubated with the secondary antibodies for $1 \mathrm{~h}$ at room temperatures. The anti-PDS5B antibody was obtained from Bethyl laboratories. Anti-PDS5B antibody, anti-tubulin antibody, and the secondary antibodies were bought from Cell Signaling Technology (Danvers, MA, USA). The bands were visualized by the Tanon5200 (Shanghai, China) and ImageJ software was used to measure the intensity of proteins.

\section{In vivo experiments}

The animal study was approved by the Animal Care and Use Committee of Bengbu Medical College. The in vivo experiments were operated following the previous description [33]. To establish the lung cancer xenografts, the H1975 cells with overexpressed PDS5B or the control cells transfected with empty vector were injected into five-week-old male nude mice $(8 \mathrm{mice} /$ group). After 2 weeks of injection, the tumor size was measured with a caliper per week. The weights of tumor masses were detected after the mice were executed at 6 weeks.

\section{Human lung cancer patient samples}

The tumor samples of lung cancer patients and their matched adjacent non-cancerous tissues were obtained from Outdo Biotech Company (Shanghai, China). The expression of PDS5B and LATS1 was determined by immunohistochemical studies in tumor specimens [27].

\section{Statistical analysis}

All results were presented as the mean \pm standard deviation. All data are representative of three independent experiments. A Student's $t$ test was used to evaluate the differences between the two groups. Analysis of variance followed by Tukey's post hoc test was used to compare the differences among multiple groups. $P<0.05$ was considered statistically significant.

\section{REFERENCES}

1. Siegel RL, Miller KD, Jemal A. Cancer statistics, 2020. CA Cancer J Clin. 2020;70:7-30.

2. Zhu T, Bao X, Chen M, Lin R, Zhuyan J, Zhen T, et al. Mechanisms and future of non-small cell lung cancer metastasis. Front Oncol. 2020;10:585284.

3. Linehan A, Forde PM. Moving immunotherapy into early-stage lung cancer. Cancer J. 2020;26:543-7.

4. Ricciuti $B$, Awad MM. What is the standard first-line treatment for advanced nonsmall cell lung cancer? Cancer J. 2020;26:485-95.

5. Conibear J. Rationale for concurrent chemoradiotherapy for patients with stage III non-small-cell lung cancer. Br J Cancer. 2020;123:10-17.

6. Passaro, A, Malapelle, U, Del Re, M, Attili, I, Russo, A, Guerini-Rocco, E et al. Understanding EGFR heterogeneity in lung cancer. ESMO Open. 20202;5: e000919.

7. Hu C, Hui K, Jiang $X$. Effects of microRNA regulation on antiangiogenic therapy resistance in non-small cell lung cancer. Biomed. Pharmacother. 2020;131:110557.

8. Ye R, Tang R, Gan S, Li R, Cheng Y, Guo L, et al. New insights into long non-coding RNAs in non-small cell lung cancer. Biomed. Pharmacother. 2020;131:110775.

9. Zhao S, Li S, Liu W, Wang Y, Li X, Zhu S, et al. Circular RNA signature in lung adenocarcinoma: a mioncocirc database-based study and literature review. Front Oncol. 2020;10:523342.

10. Wang C, Tan S, Li J, Liu WR, Peng Y, Li W. CircRNAs in lung cancer - biogenesis, function and clinical implication. Cancer Lett. 2020;492:106-15.

11. Jiang W, Xia J, Xie S, Zou R, Pan S, Wang ZW, et al. Long non-coding RNAs as a determinant of cancer drug resistance: Towards the overcoming of chemoresistance via modulation of IncRNAs. Drug Resist Updat. 2020;50:100683.

12. Lo Sardo F, Strano S, Blandino G. YAP and TAZ in lung cancer: oncogenic role and clinical targeting. Cancers (Basel). 2018;10:137.

13. Hsu PC, Yang CT, Jablons DM, You L. The crosstalk between Src and Hippo/YAP signaling pathways in non-small cell lung cancer (NSCLC). Cancers (Basel) 2020;12:1361.

14. Xie H, Wu L, Deng Z, Huo Y, Cheng Y. Emerging roles of YAP/TAZ in lung physiology and diseases. Life Sci. 2018;214:176-83.

15. Dey A, Varelas X, Guan KL. Targeting the Hippo pathway in cancer, fibrosis, wound healing and regenerative medicine. Nat. Rev. Drug Discov. 2020;19:480-94.

16. Kulkarni A, Chang MT, Vissers JHA, Dey A, Harvey KF. The hippo pathway as a driver of select human cancers. Trends Cancer. 2020;6:781-96.

17. Denes V, Pilichowska M, Makarovskiy A, Carpinito G, Geck P. Loss of a cohesinlinked suppressor APRIN (Pds5b) disrupts stem cell programs in embryonal carcinoma: an emerging cohesin role in tumor suppression. Oncogene. 2010;29:3446-52.

18. Brough R, Bajrami I, Vatcheva R, Natrajan R, Reis-Filho JS, Lord CJ, et al. APRIN is a cell cycle specific BRCA2-interacting protein required for genome integrity and a predictor of outcome after chemotherapy in breast cancer. EMBO J. 2012;31:1160-76.

19. Mo W, Zhang J, Li X, Meng D, Gao Y, Yang S, et al. Identification of novel ARtargeted microRNAs mediating androgen signalling through critical pathways to regulate cell viability in prostate cancer. PLoS ONE. 2013;8:e56592.

20. Kim MS, An CH, Yoo NJ, Lee SH. Frameshift mutations of chromosome cohesionrelated genes SGOL1 and PDS5B in gastric and colorectal cancers with high microsatellite instability. Hum Pathol. 2013;44:2234-40.

21. Blighe K, Kenny L, Patel N, Guttery DS, Page K, Gronau JH, et al. Whole genome sequence analysis suggests intratumoral heterogeneity in dissemination of breast cancer to lymph nodes. PLoS ONE. 2014;9:e115346.

22. Franca JA, Diniz MG, Bernardes VF, Costa-Silva RC, Souza RP, Gomez RS, et al. Cohesin subunits, STAG1 and STAG2, and cohesin regulatory factor, PDS5b, in oral squamous cells carcinomas. J Oral Pathol Med. 2017;46:188-93.

23. Morales $C$, Ruiz-Torres $M$, Rodriguez-Acebes $S$, Lafarga $V$, Rodriguez-Corsino $M$, Megias D, et al. PDS5 proteins are required for proper cohesin dynamics and participate in replication fork protection. J Biol Chem. 2020;295:146-57.

24. Martino J, Brunette GJ, Barroso-Gonzalez J, Moiseeva TN, Smith CM, Bakkenist CJ, et al. The human Shu complex functions with PDS5B and SPIDR to promote homologous recombination. Nucleic Acids Res. 2019;47:10151-65.

25. Couturier AM, Fleury H, Patenaude AM, Bentley VL, Rodrigue A, Coulombe $Y$, et al. Roles for APRIN (PDS5B) in homologous recombination and in ovarian cancer prediction. Nucleic Acids Res. 2016;44:10879-97. 
26. Ma J, Cao T, Cui Y, Zhang F, Shi Y, Xia J, et al. miR-223 regulates cell proliferation and invasion via targeting PDS5B in pancreatic cancer cells. Mol Ther Nucleic Acids. 2019;14:583-92.

27. Ma J, Cui Y, Cao T, Xu H, Shi Y, Xia J, et al. PDS5B regulates cell proliferation and motility via upregulation of Ptch2 in pancreatic cancer cells. Cancer Lett. 2019;460:65-74.

28. Yu J, Qin B, Moyer AM, Nowsheen S, Tu X, Dong H, et al. Regulation of sister chromatid cohesion by nuclear PD-L1. Cell Res. 2020;30:590-601.

29. Sohn MS, Kang M, Kang SM, Bae S. Downregulation of APRIN expression increases cancer cell proliferation via an interleukin-6/STAT3/cyclin D axis. Oncol Lett. 2021;21:55.

30. Shi Y, Cao T, Sun Y, Xia J, Wang P, Ma J. Nitidine chloride inhibits cell proliferation and invasion via downregulation of YAP expression in prostate cancer cells. Am J Transl Res. 2019;11:709-20.

31. Xu H, Cao T, Zhang X, Shi Y, Zhang Q, Chai S, et al. Nitidine chloride inhibits SIN1 expression in osteosarcoma cells. Mol Ther Oncolytics. 2019;12:224-34.

32. Zhang J, Wu L, Lian C, Lian S, Bao S, Wang $P$, et al. Nitidine chloride possesses anticancer property in lung cancer cells through activating Hippo signaling pathway. Cell Death Discov. 2020;6:91.

33. Ma J, Zeng F, Ma C, Pang H, Fang B, Lian C, et al. Synergistic reversal effect of epithelial-to-mesenchymal transition by miR-223 inhibitor and genistein in gemcitabine-resistant pancreatic cancer cells. Am J Cancer Res. 2016;6:1384-95

\section{ACKNOWLEDGEMENTS}

This research was supported in part by the major Project from The Key Project of Natural Science Foundation of Anhui Provincial Department of Education (KJ2019A0354) and Innovative Training Program for National University students (201910367023).

\section{COMPETING INTERESTS}

The authors declare no competing interests.

\section{ADDITIONAL INFORMATION}

Correspondence and requests for materials should be addressed to P.W., J.M. or Y.L.

Reprints and permission information is available at http://www.nature.com/ reprints

Publisher's note Springer Nature remains neutral with regard to jurisdictional claims in published maps and institutional affiliations.

Open Access This article is licensed under a Creative Commons Attribution 4.0 International License, which permits use, sharing, adaptation, distribution and reproduction in any medium or format, as long as you give appropriate credit to the original author(s) and the source, provide a link to the Creative Commons license, and indicate if changes were made. The images or other third party material in this article are included in the article's Creative Commons license, unless indicated otherwise in a credit line to the material. If material is not included in the article's Creative Commons license and your intended use is not permitted by statutory regulation or exceeds the permitted use, you will need to obtain permission directly from the copyright holder. To view a copy of this license, visit http://creativecommons. org/licenses/by/4.0\%.

(c) The Author(s) 2021 